\title{
Curvature and chirality dependence of the properties of point defects in nanotubes
}

\author{
Johan M. Carlsson* \\ Fritz-Haber-Institut der Max-Planck-Gesellschaft, Faradayweg 4-6, D-14195 Berlin, Germany.
}

Received 7 September 2006, accepted 15 September 2006

Published online 25 October 2006

Key words Nanotubes, Point defects, DFT calculations.

PACS 61.72.Ji, 73.22.-f, 81.07.De

This article presents a systematic study of how point defects, such as SW-defects and vacancies, influence the properties of nanotubes. The DFT calculations show that large atomic relaxations at vacancies leads to a contraction of the nanotube. The formation energy $E_{\text {form }}$, has a curvature, chirality and a family dependence, where $E_{\text {form }}$ is slightly lower in metallic compared to semiconducting nanotubes. Vacancies become electrically active due to defect states close to $\epsilon_{\mathrm{F}}$ and its population depend on the chirality and the position of $\epsilon_{\mathrm{F}}$. A model based on the heat of formation of defective nanotubes furthermore provide an upper estimate for the defect concentration, which is in better agreement with values from AFM experiments than the standard equilibrium approximation.

Copyright line will be provided by the publisher

\section{Introduction}

Carbon nanotubes (CNT) have attracted a tremendous attention ever since their discovery. The interest is in particular connected to the extraordinary properties of perfect tubes. However, atomic force microscopy measurements (AFM) [1], indicate that the average defect concentration is much higher than theoretical equilibrium estimates. These defects may on one hand have adverse effects on the performance, but their presence can, on the other hand, also give rise to new phenomena such as an all-carbon quantum dot [2]. Point defects can be observed by scanning tunneling microscopy (STM) as they give rise to a local increase in the signal. Scanning tunneling spectroscopy (STS) reveals that this is due to localized defect states with an energy close to the Fermi level, $\epsilon_{\mathrm{F}}$ [3]. Transmission electron microscopy (TEM) [4, 5] has shown a curvature dependence of the formation energy during electron irradiation experiments, but determining the exact type and orientation of a point defect with respect to the tube axis is still difficult. A connection between theory and experiments is therefore necessary for an unambiguous classification [6, 7]. Individual point defects, such as adatoms [8], topological defects like the Stone-Wales defect (SW) [9], single vacancies [10,11], double vacancies [12] and vacancy-interstitial complexes [13], have been studied by tight-binding (TB) and density-functional (DFT) calculations in order to complement the experimental information. However, a complete theory for point defects in nanotubes is still not available.

\section{Methods}

The calculations are based on the density-functional theory (DFT) in the generalized gradient approximation for the exchange-correlation functional [14] as implemented in the Castep [15] and DMol ${ }^{3}$-codes [16]. $\mathrm{DMol}^{3}$ is an all-electron code with atom-centered, numerical basis functions cut-off at 12 bohr, while the

\footnotetext{
* Corresponding author: e-mail: johanc@fhi-berlin.mpg.de, Phone: +49308413 4835, Fax: +49 3084134701
} 

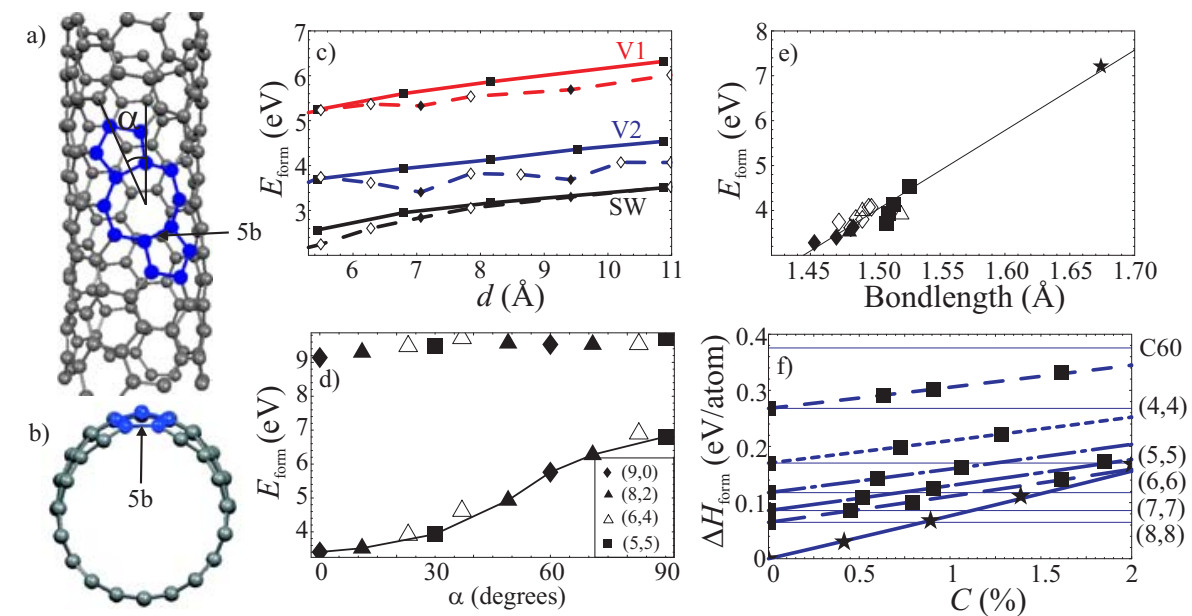

Fig. 1 (online color at www.pss-b.com) (a) An (8,2)-nanotube with a V2 vacancy. The non-hexagonal rings are highlighted in blue and the misorientation angle $\alpha$ between the mirror plane and the tube axis is indicated. $5 \mathrm{~b}$ shows the pentagon bond. b) A cut through a (10,0)-nanotube indicating the contraction at the V2-vacancy due to the relaxation. c) Formation energies, $E_{\text {form }}$, for SW-defect and vacancies V1-V2 as function of nanotube diameter $d$. Solid lines with boxes show armchair nanotubes and dashed lines with diamonds zigzag nanotubes. Filled symbols for metallic and open symbols for semiconducting SWCNT. d) $E_{\mathrm{form}}$ as function of the misorientation angle $\alpha$ as is indicated in a). The upper row of symbols are $E_{\text {form }}$ before relaxation and the lower row are fully relaxed values. The values for the metallic nanotubes are joined by a line to guide the eye. e) $E_{\text {form }}$ as function of pentagon bond length for the V2 vacancy in different SWCNTs indicating the linear dependence of $E_{\mathrm{form}}$ of $d$. Solid symbols indicate metallic nanotubes and open symbols semiconducting nanotubes. The star indicates $E_{\text {form }}$ in graphene. f) Heat of formation $\Delta H$, of graphene and armchair nanotubes as function of defect concentration $C$. The horizontal lines indicate $\Delta H$ for the perfect nanotubes and C60.

Castep-code uses ultrasoft pseudopotentials and a plane-wave basis set with an energy cut-off of $450 \mathrm{eV}$. The nanotubes were modeled in a supercell with periodic boundary conditions and were separated by at least $12 \AA$ of vacuum to avoid tube-tube interactions. The supercells were constructed to have a similar length for all studied SWCNTs, such that the defect separation along the tube axis in all nanotubes was around $17 \AA$. The perfect nanotubes contained up to 224 atoms and all atoms were fully relaxed. The Brillouin zone was sampled at $6 k$-points along the tube axis. The formation energy of a defect in a $(\mathrm{n}, \mathrm{m})$ nanotube is:

$$
E_{\text {form }}\left(N_{\mathrm{C}}, \mu_{(\mathrm{n}, \mathrm{m})}\right)=\left\{E_{(\mathrm{n}, \mathrm{m})}^{\mathrm{Def}}+N_{\mathrm{C}} \mu_{(\mathrm{n}, \mathrm{m})}\right\}-E_{(\mathrm{n}, \mathrm{m})}+q \epsilon_{\mathrm{F}}
$$

where $E_{(\mathrm{n}, \mathrm{m})}$ and $E_{(\mathrm{n}, \mathrm{m})}^{\text {Def }}$ refer to the total energy of a $(\mathrm{n}, \mathrm{m})$ nanotube before and after the defect is created. $\mu_{(\mathrm{n}, \mathrm{m})}$ denotes the chemical potential of an atom in this nanotube and $N_{\mathrm{C}}$ is the number of removed atoms needed to form the defect. $q$ refers to the charge state of the defect and $\epsilon_{\mathrm{F}}$ is the Fermi level in the system. A jellium background was used to compensate the charge of the additional electrons during charged calculations.

\section{Results and discussion}

Electron-irradiation experiments have shown that vacancies are preferentially formed in the inner tubes of multiwalled nanotubes, MWCNT, indicating that the formation energy decreases in smaller nanotubes [4, 5]. Fig. 1c) shows accordingly that the formation energy of SW-defects and single (V1) and double (V2) 

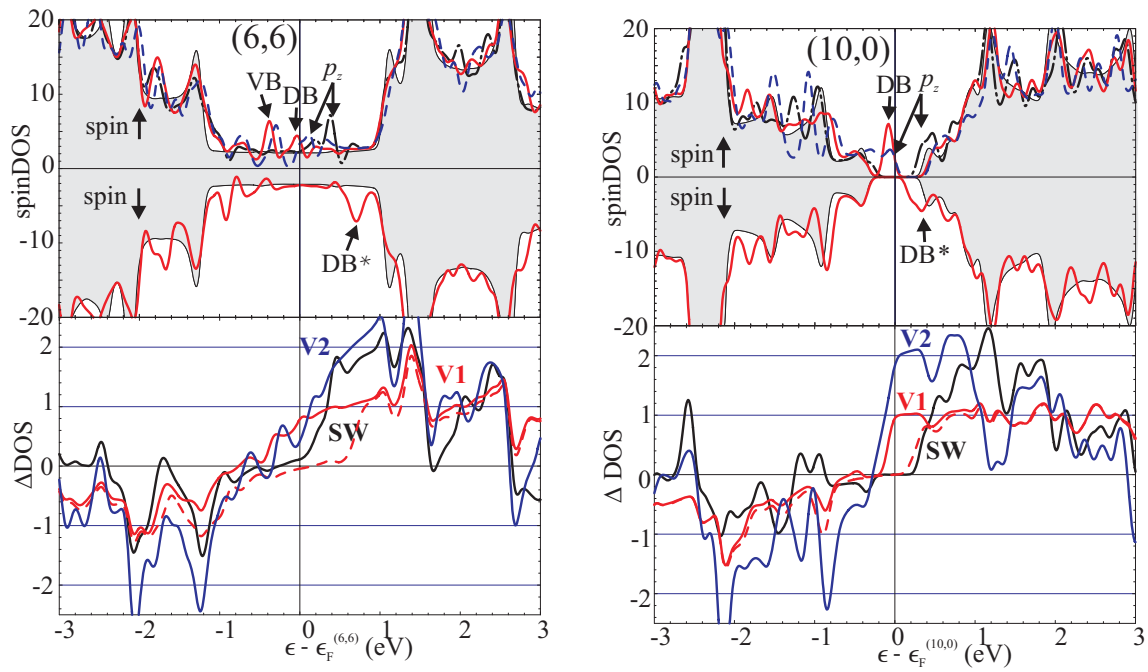

Fig. 2 (online colour at www.pss-b.com) Upper panel: Spin polarized density of states (spinDOS) for a metallic $(6,6)$ and a semiconducting $(10,0)$ nanotube with a SW-defect (black dashed line), single vacancy, V1, (red solid line) and divacancy V2, (blue dash-dotted line). The gray shaded regions show the DOS for the pristine nanotube and the energy scale is set to zero at the corresponding $\epsilon_{\mathrm{F}}^{(\mathrm{n}, \mathrm{m})}$, indicated by the vertical line. The arrow marked DB shows the position of the dangling bond state, VB indicate the position of the valence band in the $(6,6)$ nanotube and $p_{\mathrm{z}}$ shows the position of the $p_{\mathrm{z}}$-defect state. Lower panel: the integrated difference DOS ( $\left.\triangle \mathrm{DOS}\right)$ for the same defects.

vacancies generally decreases as function of curvature in agreement with previous tight-binding $[9,10]$ and DFT calculations [12]. This is particularly evident for the armchair SWCNT, where the formation energy is inversely proportional to the diameter as shown in Fig. 1c). The formation energy for the corresponding point defects is lower in zigzag SWCNT indicating that there is also a chiral dependence of the formation energy. Varying the misorientation angle $\alpha$ for $\mathrm{V} 2$ in a set of metallic nanotubes with similar diameter in Fig. 1d) shows that the formation energy is lower, the smaller the value of $\alpha$. However, the V2 cannot form with an arbitrary angle, since the orientation is determined by the lattice of the nanotube. $\alpha$ is therefore determined by the chiral angle $\theta$, because the smallest formation energy for V2 occurs for $\alpha=\theta_{(n, m)}$.

Fig. 1e) shows that $E_{\text {form }}$ is basically linearly dependent on the bond length of the pentagon bonds for $\mathrm{V} 2$ in agreement with the linear coupling theory for lattice distortions at defects [17]. The curvature and chiral dependencies therefore originate from the improved relaxation of the atoms surrounding the point defects as can be seen by comparing the formation energy of relaxed and unrelaxed V2 in Fig. 1d). The relaxation is particularly important for the undercoordinated atoms that are sitting around a vacancy. These atoms tend to rebind with each other in order to reconstruct incomplete hexagons into pentagons and the relaxation in addition forms a large ring surrounding the vacancy as can be seen for the V2 in Fig. 1a). The vacancies can then be divided into even and odd [18], where the even take the form of a topological defect, while odd vacancies have one remaining undercoordinated atom. The curvature facilitates the relaxation of the pentagon bonds, since the atoms can move inwards and thereby shorten the bond distance as can be seen in Fig. 1b). The relaxation consequently leads to a contraction of the nanotube at vacancies in agreement with the observation that nanotubes shrink during electron irradiation due to the loss of atoms and formation of vacancies in the lattice $[4,5]$.

Fig. 1c) furthermore shows that the formation energy for defects in zigzag nanotubes has a periodic behavior, since the metallic zigzag-tubes have lower formation energy than the semiconducting zigzagtubes, as has been pointed out for V1 and V2 vacancies previously [10, 12]. However, Fig. 1d) reveals that 
this is a general behavior, since the formation energy for V2 in a semiconducting $(6,4)$-SWCNT is slightly higher than in the corresponding metallic SWCNTs. This family behavior originates from the fact that the states in graphene become less and less bonding as they approach the $K$-point in the Brillouin zone as indicated by the negative strain-charge relationship in graphene and nanotubes [19]. The semiconducting $(n-m) \bmod 3= \pm 1$ nanotubes that omit the $K$-point are therefore marginally stiffer than the metallic, $(n-m) \bmod 3=0$ nanotubes of corresponding diameter, such that the relaxation of the pentagon bonds is slightly more favorable in metallic nanotubes leading to a relative reduction of the formation energy.

The formation energy can be used to estimate the defect concentration, but the values in Fig. 1 are very large, such that the process of creation and annihilation of defects in equilibrium is completely negligible in nanotubes at normal temperatures. The equilibrium defect separation for $\mathrm{V} 2$ in a nanotube with a diameter around $\mathrm{d}=1 \mathrm{~nm}$, such as a $(7,7)$-SWCNT, would be around $50 \mu \mathrm{m}$ even at $3000{ }^{\circ} \mathrm{C}$. AFM measurements show on the other hand that SWCNTs produced by arc-discharge have an average defect separation of about $350 \mathrm{~nm}$ [1]. This indicates that the kinetics of the synthesis and the purification process determine the defect concentration in nanotubes [20]. It may therefore be more appropriate to compare the heat of formation $H_{\text {form }}^{(n, m)}$, of defective nanotubes in order to estimate the relative stability as function of defect concentration. A model for the $H_{\text {form }}^{(\mathrm{n}, \mathrm{m})}$ of a nanotube with defects can be formulated as:

$$
H_{\text {form }}^{(n, m)}\left(C, E_{\text {form }}\right)=H_{\text {form }}^{\mathrm{G}}+\Delta E_{(n, m)}+\frac{1}{1-\sum_{i} C_{i} N_{\mathrm{C}}^{i}} \sum_{j} C_{j} E_{\text {form }}^{j}
$$

where $H_{\text {form }}^{\mathrm{G}}$ is the heat of formation for graphene, $\Delta E_{(n, m)}=D / d_{(n, m)}^{2}$ is the curvature energy, $C_{j}$ is the concentration of the point defect with formation energy $E_{\text {form }}^{j}$, that needs $N_{\mathrm{C}}$ atoms to form. Fig. 1f) shows that the agreement is excellent between the model and the DFT values for low defect concentrations, where the defect-defect interaction is negligible. The model reveals that the change in $H_{\text {form }}^{(\mathrm{n}, \mathrm{m})}$ due to the presence of a limited amount of defects is small compared to the curvature energy. Fig. 1f) furthermore shows that each $(n, m)$-nanotube has an energy window where it has the lowest defect concentration. Nanotubes with a smaller diameter cannot form and nanotubes with a larger diameter have a lower curvature energy and consequently a higher defect concentration to have the same $H_{\text {form }}^{(\mathrm{n}, \mathrm{m})}$. The defect concentration can therefore be estimated by equating $H_{\text {form }}^{(\mathrm{n}, \mathrm{m})}$ for a defective nanotube to a perfect nanotube with similar diameter. Comparing $H_{\text {form }}^{(\mathrm{n}, \mathrm{m})}$ for a $(7,7)$ to a $(11,2)$-SWCNT that has more or less the same diameter, suggests that a (7,7)-SWCNT could have a defect separation of V2 down to $50 \mathrm{~nm}$. This appears to be a reasonable upper estimate within an order of magnitude from the AFM measurements.

Point defects have a strong influence on the electronic properties of nanotubes. Vacancies open up a local band gap in armchair SWCNT, due to an avoided band crossing of the $\pi$ - and $\pi^{*}$-band. This can be seen as a small dip in the DOS of the $\pi$-band (marked VB) for the $(6,6)$-SWCNT close to $\epsilon_{\mathrm{F}}$ in Fig. 2 . The point defects also lead to the formation of a $p_{z}$-defect state and undercoordinated atoms at odd vacancies have a dangling bond as exemplified by the V1 vacancy in Fig. 2. The wavefunction for the $p_{z}$-defect state is a combination of $p_{z}$-orbitals surrounding the defect and it has bonding character along the pentagon bond as illustrated by V2 in Fig. 3. The curvature hybridizes the wavefunctions, which increases the mixing between the $p_{z}$ defect states and the $\pi$-band. The mixing is stronger the smaller the band gap, such that the $p_{z}$-defect states become more delocalized in metallic nanotubes with small diameter as can be seen by comparing the wavefunctions of V2 in the armchair-SWCNT in Fig. 3. The energy of the $p_{z}$-defect state is close to the intrinsic value of $\epsilon_{\mathrm{F}}^{(n, m)}$, such that it appears as a peak in the band gap of the $(10,0)$ - and $(6,6)$-SWCNTs. This is in agreement with STS measurements that show additional states between the van-Hove singularities at defect sites in SWCNT [3]. The $\triangle$ DOS in Fig. 2 suggests that the $p_{z}$-state at the SW-defect remains unoccupied even for large variations of $\epsilon_{\mathrm{F}}$, while the V1 and V2 may be occupied depending on the exact position of $\epsilon_{\mathrm{F}}$. A charge state analysis demonstrates that the $\epsilon(0 /-1)$ and $\epsilon(-1 /-2)$ transition for the V2 in a $(10,0)$-SWCNT occur for $\epsilon_{\mathrm{F}}$ at the bottom of the bandgap and close to middle of the bandgap. The V2 in a $(6,6)$-SWCNT in contrast has the $\epsilon(0 /-2)$ transition just above the 
$(8,8)$

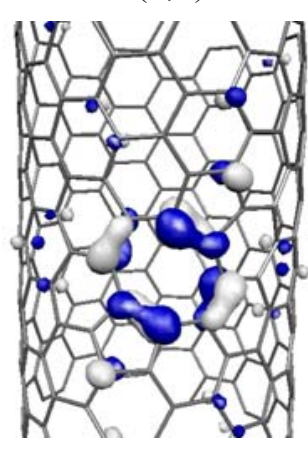

$(6,6)$

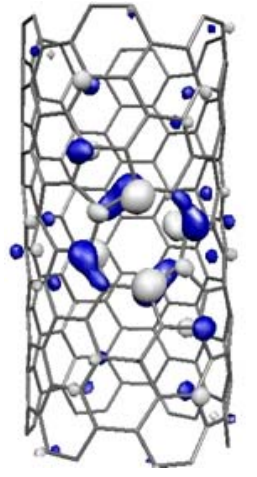

$(4,4)$

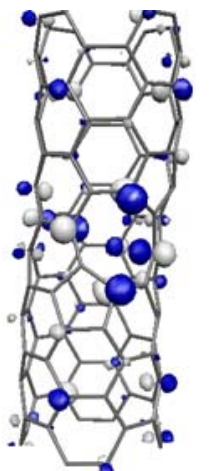

Fig. 3 (online colour at www.pssb.com) Wave functions for the $p_{z}$ defect state at the V2 vacancy in three armchair nanotubes. Note how the wavefunction becomes more delocalized as the curvature increase from the $(8,8)$ - to the $(4,4)$-nanotube.

intrinsic position of $\epsilon_{\mathrm{F}}^{(6,6)}$. The different behavior of the $p_{z}$-state in the (10,0)- compared to (6,6)-SWCNT indicate that the energy of the $p_{z}$-state has a chiral dependence that can be related to the shorter bondlength for the pentagon bond in the (10,0)-SWCNT. Occupation of the $p_{z}$-state stabilizes V2, since the pentagon bond is shorter and the formation energy is $0.5 \mathrm{eV}$ lower for the V2 in a $(10,0)$-SWCNT in the $q=-2$ compared to the $q=0$ charge state when $\epsilon_{\mathrm{F}}$ is in the middle of the bandgap. The largest stabilization can occur for vacancies in semiconducting nanotubes, where the $p_{z}$-defect state remains relatively localized and $\epsilon_{\mathrm{F}}$ can be manipulated. This furthermore indicates that it is necessary to consider different charge states to obtain the proper formation energy for point defects in nanotubes.

\section{Conclusions}

The DFT calculations show that the properties of the point defects and the nanotubes are interrelated. The formation energy has a curvature dependence and the model for the heat of formation indicates that the concentration of point defects increases, the smaller the diameter of the nanotube. The formation energy also has a chiral and a family dependence, such that the defect concentration may be larger in metallic nanotubes. However, the point defects induce defect states and the occupation of the $p_{z}$-state may lead to a local charging of the point defect. The occupation of the $p_{z}$-state stabilizes the point defect and the strongest effect appears in semiconducting nanotubes, which tend to compensate for the family effect. The defect concentration may therefore be just slightly larger in metallic nanotubes and the point defect may have different electronic character depending on the nanotube. The properties of defective nanotubes are therefore very sensitive to both the properties of the nanotube and the type, the concentration and the orientation of the point defects that are present in the nanotube.

Acknowledgements The author would like to thank Prof. Scheffler for useful discussions.

\section{References}

[1] Y. Fan, M. Burghard, and K. Kern, Adv. Mat. 14130 (2002).

[2] M. Bockrath, W. Liang, D. Bozovic, J. H. Hafner et al., Science 291, 283 (2001).

[3] M. Ouyang, J.-L. Huang, and C. M. Lieber, Phys. Rev. Lett. 88, 066804 (2002).

[4] P. M. Ajayan, V. Ravikumar, and J.-C. Charlier, Phys. Rev. Lett. 81, 1437 (1998).

[5] F. Banhart, J. X. Li, and A. V. Krasheninnikov, Phys. Rev. B 71, 241408 (2005).

[6] M. Ishigami, H. J. Choi, S. Alouni, S. G. Louie et al., Phys. Rev. Lett. 93, 196803 (2004).

[7] S. Lee, G. Kim, B.-Y. Choi, J. Lee et al., Phys. Rev. Lett. 95, 166402 (2005).

[8] A. V. Krasheninnikov, K. Nordlund, P. O. Lehtinen, A. S. Foster et al., Phys. Rev. B 69, 073402 (2004).

[9] B. C. Pan, W. S. Yang, and J. Yang, Phys. Rev. B 62, 12652 (2000).

[10] A. J. Lu and B. C. Pan, Phys. Rev. Lett. 92, 105504 (2004). 
[11] S. B. Fagan, L. B. da Silva, and R. Mota, Nano Lett. 3, 289 (2003).

[12] A. V. Krasheninnikov, P. O. Lehtinen, A. S. Foster, and R. M. Nieminen, Chem. Phys. Lett. 418, 132 (2006).

[13] A. J. R. da Silva, A. Fazzio, and A. Antonelli, Nano Lett. 5, 1045 (2005).

[14] J. P. Perdew, K. Burke, and M. Ernzerhof, Phys. Rev. Lett. 77, 3865 (1996).

[15] CASTEP, M. D. Segall, P. J. D. Lindan, M. J. Probert, C. J. Pickard et al., J. Phys.: Cond. Matt. 14, 2717 (2002).

[16] $\mathrm{DMol}^{3}$, Academic version: B. Delley, J. Chem. Phys. 92, 508 (1990).

[17] A. M. Stoneham, Theory of Defects in Solids, (Oxford University Press, Oxford, UK, 1975) chap. 8.

[18] J. M. Carlsson and M. Scheffler, Phys. Rev. Lett 96, 046806 (2006).

[19] G. Sun, J. Kürti, M. Kertesz, and R. Baughman, J. Am. Chem. Soc. 124, 15076 (2002).

[20] U. J. Kim, C. A. Furtado, X. Liu, G. Chen et al., J. Am. Chem. Soc. 127, 15437 (2005). 